\title{
ClaimPortal: Integrated Monitoring, Searching, Checking, and Analytics of Factual Claims on Twitter
}

\author{
Sarthak Majithia Fatma Arslan Sumeet Lubal \\ Damian Jimenez Priyank Arora Josue Caraballo Chengkai Li \\ Department of Computer Science and Engineering \\ The University of Texas at Arlington
}

\begin{abstract}
We present ClaimPortal, a web-based platform for monitoring, searching, checking, and analyzing English factual claims on Twitter. We explain the architecture of ClaimPortal, its components and functions, and the user interface. While the last several years have witnessed a substantial growth in interests and efforts in the area of computational factchecking, ClaimPortal is a novel infrastructure in that fact-checkers have largely skipped factual claims in tweets. It can be a highly powerful tool to both general web users and factcheckers. It will also be an educational resource in helping cultivate a society that is less susceptible to falsehoods. While it currently focuses on politics-related tweets, it will be extended to include more general factual claims.
\end{abstract}

\section{Introduction}

The spreading of falsehoods on the web has adverse effects on a myriad of aspects in our society. Politicians are doubling down on claims that are demonstrably false because of the safety net that "fake news" affords them. These efforts to manipulate and distort public opinions in order to gain political leverage can have negative effects on a democracy, and they can even result in the potential manipulation of democratic election results.

At news organizations such as The Washington Post, New York Times and FactCheck.org, professional fact-checkers take on the hard battle to counter misinformation and disinformation. They vet claims by analyzing relevant data and documents and publishing their verdicts. For instance, PolitiFact.com gives factual claims truthfulness ratings such as true, half true, false, and even "pants on fire". However, there is simply far more misinformation on the web than what factcheckers can keep up with. The process of factchecking is laborious and intellectually demand- ing, as it takes the professionals about one day to research and write a typical article about a factual claim (Hassan et al., 2015a). This difficulty leaves many harmful claims unchecked, since factchecking organizations can only use their limited resources to focus on national events and prominent figures.

This problem of unchecked claims is exacerbated on social media. On the one hand, it is unlikely fact-checkers are able to check every social media post, due to limited resources and the sheer volume of data. ${ }^{1}$ On the other hand, a large number of false claims, likely much more than those in traditional media, are being spread through social media. This can be due to the compounded effect of several factors: social media platforms have become increasingly important to public figures and organizations in engaging with voters and citizens; mobile devices have brought an age in which sharing and disseminating information is easy for anyone, including both malicious and unintentional creators of falsehoods; the falsehoods are further replicated and amplified by social media bots and clickbait articles. The consequence can be devastating. For instance, a recent study reports that a sample of 140,000 Twitter users in the battleground state of Michigan shared as many junk news items as professional news during the final ten days of the 2016 election, each constituting $23 \%$ of the web links they shared on Twitter in that period. ${ }^{2}$

In this paper we present ClaimPortal, a webbased platform for monitoring, searching, checking, and analytics of factual claims on Twitter. ClaimPortal is available at https://idir. uta.edu/claimportal. ClaimPortal continuously collects tweets and monitors factual

\footnotetext{
${ }^{1}$ https://mashable.com/article/ snopes-stops-fact-checking-for-facebook/

${ }^{2}$ http://politicalbots.org/?p=1064
} 
claims embedded in tweets. It is integrated with fact-checking tools, including a claim matcher which finds known fact-checks matching any given tweet, a claim spotter which scores each claim and the corresponding tweet based on their check-worthiness, i.e., how important it is to factcheck them. ClaimPortal provides an intuitive and convenient search interface that assists its users to sift through these factual claims in tweets using filtering conditions on dates, twitter accounts, content, hashtags, check-worthiness scores, and types of claims. ClaimPortal also provides simple analytics and visualization tools for discovering patterns pertinent to how certain twitter accounts make claims, how different types of claims are distributed, and so on.

The initial call to arms to research on computational fact-checking was made nearly a decade ago (Cohen et al., 2011). The last several years have witnessed a substantial growth in interests and efforts in this arena. These efforts tackle various fronts, from detecting important factual claims that are worth checking (Hassan et al., 2015b; Jimenez and $\mathrm{Li}, 2018$ ), to using databases for discerning factual claims' robustness (Wu et al., 2017) and truthfulness (Ciampaglia et al., 2015; Shi and Weninger, 2016; Jo et al., 2019), to building end-to-end fact-checking systems (Babakar and Moy, 2016; Hassan et al., 2017a,b), and visualizing the spread of claims (Shao et al., 2016). ClaimPortal is a novel infrastructure in that factcheckers have largely skipped factual claims in tweets, especially those from less prominent accounts, due to limited resources.

\section{System Architecture and Components}

\subsection{System Architecture}

ClaimPortal is composed of a front-end web based GUI, a MySQL database, an Elasticsearch ${ }^{3}$ search engine, an API, and several decoupled batch data processing components (Figure 1). The system operates on two layers. The front-end presentation layer allows users to narrow down search results by applying multiple filters. Keyword search on tweets is powered by Elasticsearch which is coupled with querying the database to provide additional filters. Additionally, it provides numerous visualized graphs. The back-end data collection and computation layer performs pre-processing

\footnotetext{
${ }^{3}$ https://www.elastic.co/products/ elasticsearch
}

of tweets, computing check-worthiness scores of tweets using the public ClaimBuster API (Hassan et al., 2017a), Elasticsearch batch insertion, detecting claim types of tweets, and finding similar fact-checked claims for each tweet, using ClaimBuster API. ClaimPortal stays up-to-date with current tweets by periodically calling the Twitter REST API.

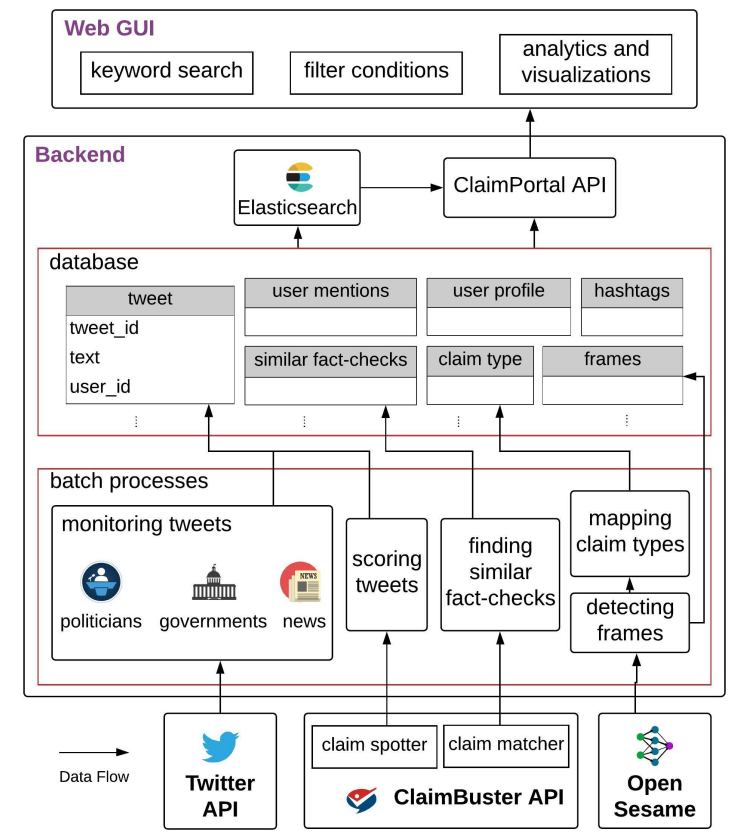

Figure 1: ClaimPortal system architecture.

\subsection{Monitoring, Processing, and Storing Tweets}

ClaimPortal at this moment focuses on politicallycharged tweets, but will be expanded to eventually cover all types of tweets. We curated a list of prominent Tweet handles in U.S. politics that include but are not limited to house representatives and senators in the Congress, governors, city mayors, U.S. Cabinet members, other government officials, and political teams of news media. We then made use of the user_timeline endpoint of the Twitter REST API to navigate through each user's timeline and collected their tweets. More specifically, we navigated through the historic data of a user's timeline, which is a one-time process. We then keep our data up-to-date by continuously monitoring newly posted tweets. As of April 10, 2019, ClaimPortal monitors 3,200 Twitter handles and has collected approximately 3.3 million tweets after being deployed in mid-January 2019. We are working on substantially expanding the curated list of Twitter handles. 


\begin{tabular}{|c|l|}
\hline Claim Type & \multicolumn{1}{c|}{ FrameNet Frames } \\
\hline Conflict & Invading, Attack, Explosion, Destroying, Hostile encounter, Use firearm, Shoot projectiles, Downing, Protest, Political actions \\
\hline Life & Giving birth, Being born, Death, Killing, Forming relationships, Cause harm, Personal relationship, Dead or alive \\
\hline Movement & Self motion, Inhibit movement, Travel, Departing, Arriving, Visiting, Motion, Cause motion, Bringing \\
\hline Transaction & Import export scenario, Commerce buy, Commerce sell, Getting, Commerce pay, Borrowing, Giving \\
\hline Business & Activity start, Conquering, Endeavor failure, Intentionally create, Business closure, Locale closure \\
\hline Contact & Meet with, Discussion, Come together, Communication, Contacting, Communication means, Text creation, Request \\
\hline Personnel & Take place of, Get a job, Hiring, Appointing, Removing, Firing, Quitting, Choosing, Becoming a member, Change of leadership \\
\hline Justice & $\begin{array}{l}\text { Arrest, Imprisonment, Detaining, Extradition, Breaking out captive, Try defendant, Pardon, Appeal, Verdict, Sentencing, Fining, Execution, } \\
\text { Releasing, Notification of charges }\end{array}$ \\
\hline Comparison & Comparing two entities, Comparing at two different points in time \\
\hline Quantity & Change position on scale, Creating, Causation, Cause change of position on a scale, Occupy rank, Ratio \\
\hline Stance & Taking sides, Opinion, Be in agreement on assessment, Vote, Oppose and Support Consistency \\
\hline Speech & Statement, Affirm or deny, Telling \\
\hline
\end{tabular}

Table 1: Claim types and their corresponding FrameNet frames. Frames in red color are created by us.

ClaimPortal's back-end layer focuses on data processing and storage. The Twitter REST API provides us with the necessary data. However, the system does not require all of it. In fact, a lot of the API's response is discarded to keep our database small and yet sufficient enough to provide all necessary information for the portal. This is achieved through the ClaimPortal API. The API is a web service designed using Python and the Flask ${ }^{4}$ micro-framework. It provides end points for loading tweets on the GUI, search for hashtags, and search for users in applying from-user and user-mention filters. Based on the keyword search and filters requested by a user, the API queries the database to find the resulting list of tweet IDs and returns the list as a JSON response. A tweet ID is a unique number assigned to a tweet by Twitter. By using Twitter's card API ${ }^{5}$ the system dynamically populates the latest activity of a tweet at the front-end, based on its ID.

The MySQL database has several normalized tables. For each tweet the database stores its text, when it was created, and who tweeted it. The database also stores information about re-tweets and quoted-tweets, hashtags and URLs mentioned in the tweets, and information about the accounts mentioned in the tweets.

ClaimPortal uses Elasticsearch to support keyword search over the stored tweets. Since Elasticsearch is equipped with incremental indexing, the system periodically feeds Elasticsearch the delta tweets since last update for indexing. For this the system uses a decoupled background batch process that takes care of incrementally inserting tweets and updating the Elasticsearch index.

\footnotetext{
${ }^{4}$ http://flask.pocoo.org

${ }^{5}$ https://developer.twitter.com/en/ docs/tweets/optimize-with-cards
}

\subsection{Claim Spotter}

In ClaimPortal, each tweet is given a checkworthiness score which denotes whether the tweet has a factual claim of which the truthfulness is important to the public. This score is obtained by probing the ClaimBuster API, ${ }^{6}$ a well-known factchecking tool, developed by our research group, that is being used by professional fact-checkers on a regular basis (Adair et al., 2019). ClaimBuster (Hassan et al., 2017a; Jimenez and Li, 2018) is a classification and ranking model trained on a human-labeled dataset of 8,000 sentences from past U.S. presidential debates. The ClaimBuster API returns a check-worthiness score for any given text. The score is on a scale from 0 to 1 , ranging from least check-worthy to most checkworthy. The background task of probing ClaimBuster API for getting scores for tweets is another batch process, in parallel with the tweet collection and the Elasticsearch indexing processes.

\subsection{Detecting Claim Types}

ClaimPortal uses tweets to gain insights into factual claims that are being spread, by whom, how often, and whether they are true. To answer these questions we categorize tweets by the types of factual claims they promote. We employed a collection of FrameNet frames (Baker et al., 1998) and created several new frames specifically for factual claims. We then adopted the study of mapping frames to event types (Spiliopoulou et al., 2017).

\subsubsection{Frame detection}

FrameNet is a linguistic resource for English comprised of 1,224 manually established semantic frames. Each frame provides information about both the linguistic and the semantic structure of a type of event, situation, object, or relation along with its participants. The participants, called

\footnotetext{
${ }^{6}$ https://idir.uta.edu/claimbuster/
} 


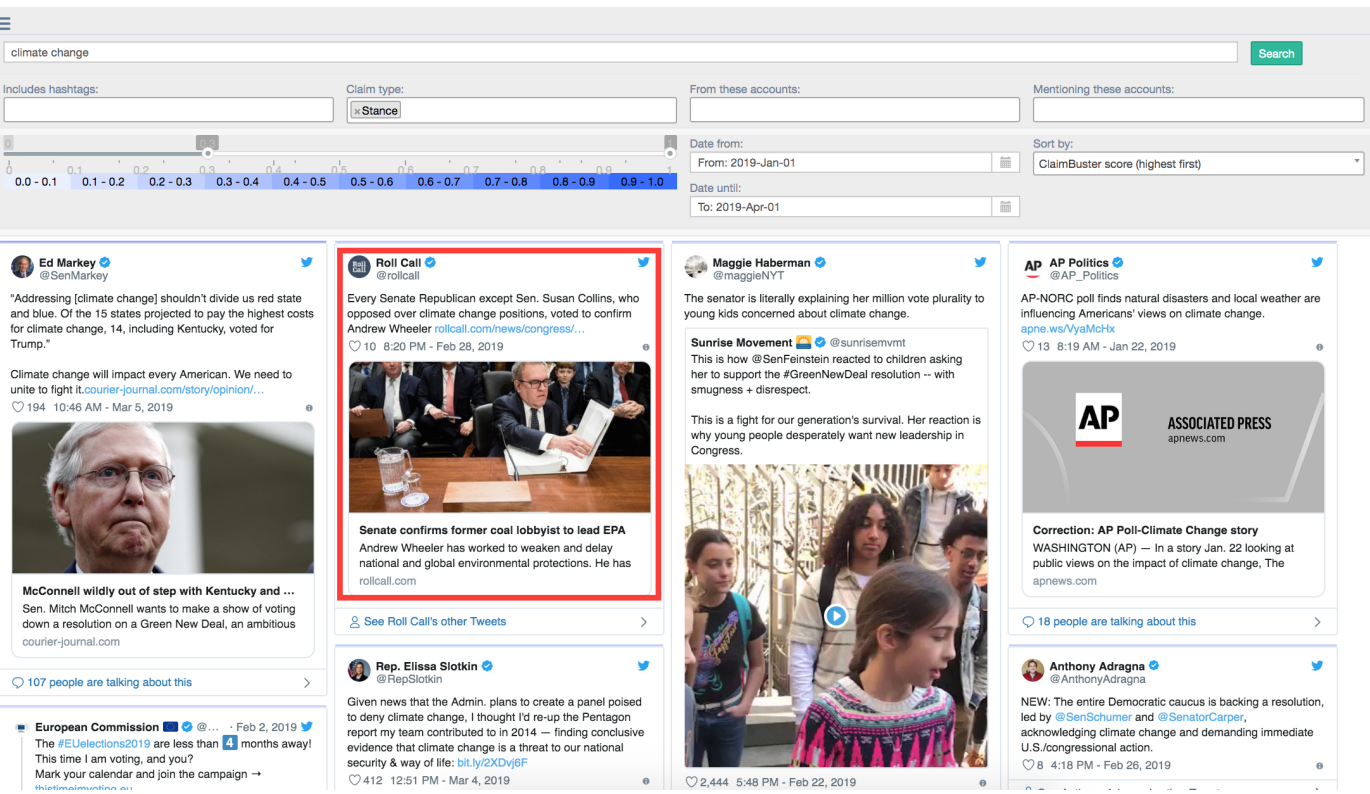

(a)

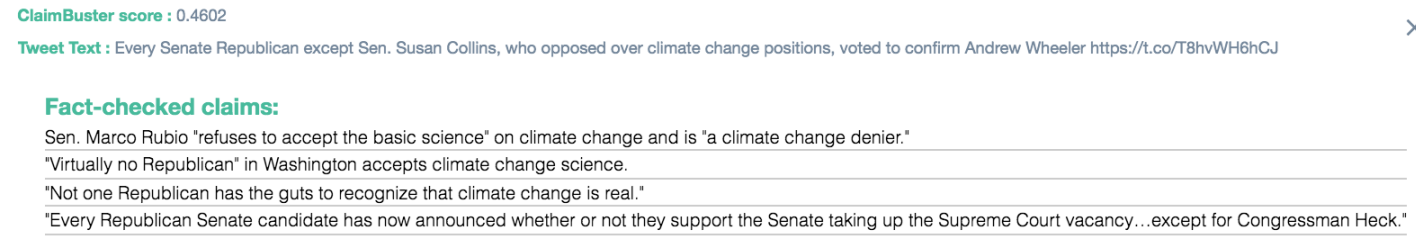

(b)

Figure 2: (a) ClaimPortal user interface. (b) Similar fact-checks for the highlighted tweet in Figure (a).

frame elements, are frame-specific semantic roles that provide additional information. Each frame is evoked by a set of lexical units, or words, which are a composition of the lemma and meaning of the word.

We created new frames after conducting a survey of existing fact-checks from PolitiFact ${ }^{7}$ and followed it by grouping together semantically and syntactically similar factual claims from these fact-checks. If a group of claims did not share a common existing frame, we created a new frame for it. Details of these purposely created new frames can be found in (Arslan et al., 2019). The corpus of the newly-defined frames along with their annotated exemplary sentences is publicly available. ${ }^{8}$

We used open-sesame (Swayamdipta et al., 2017), a recurrent neural network based frame- semantic parser, to detect all possible frames a tweet can potentially hold. We retrained open-sesame on FrameNet 1.7 dataset after extending it with annotated sentences for the newly defined frames.

\footnotetext{
${ }^{7}$ https: //www.politifact.com

${ }^{8}$ https://github.com/idirlab/factframe
}

Open-sesame works as a pipeline of several tasks: target identification (detecting all lexical units), frame identification (detecting all frames in a sentence), and argument identification.

\subsubsection{Claim type mapper}

In (Spiliopoulou et al., 2017) eight ACE event types were listed along with their mapped frames: Business, Conflict, Contact, Justice, Life, Movement, Personnel, and Transaction. To accommodate the new frames explained in Section 2.4.1, we extended this list by introducing four new event types, namely Comparison, Quantity, Stance, and Speech, and their corresponding frames (Table 1). In ensuing discussion, we refer to these event types as claim types, for simplicity of terminology. More specifically, Comparison is for claims that show entities involved in some sort of comparisons based on some criteria, Quantity presents claims with quantities, Stance is for claims that have entities with viewpoints towards issues, events, etc., and Speech is for claims that communicate some messages in the written or spoken form. A script identifies the claim types of each tweet by mapping identified frames to their corresponding claim 


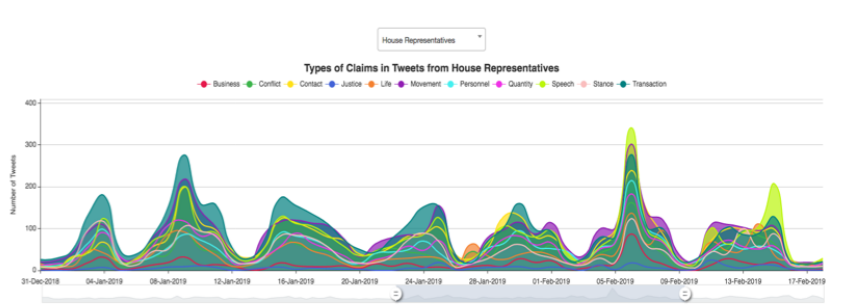

(a)

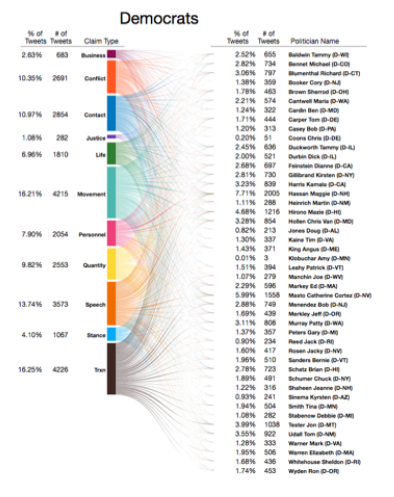

(d)

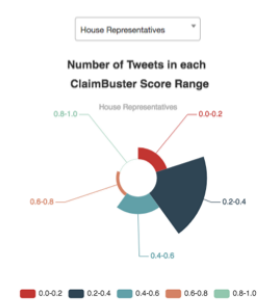

(b)

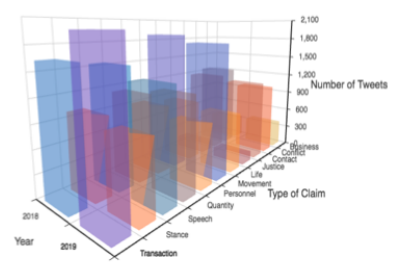

(c)

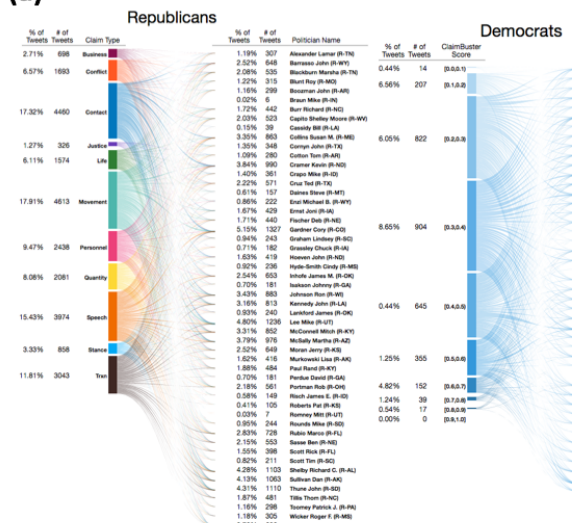

(b)

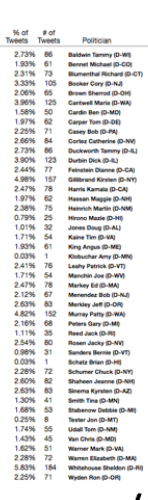

(e)

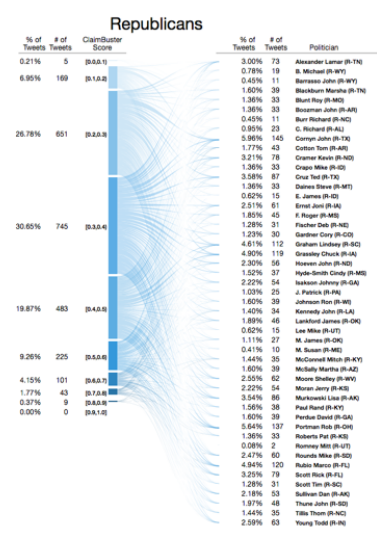

Figure 3: Examples of visualizations on ClaimPortal website.

types. A tweet can have multiple claim types.

\subsection{Claim Matcher}

Claim matching is an important step in the workflow of fact-checking. Given a factual claim, it aims at finding identical or similar claims from a repository of existing fact-checks. The premise is that public figures keep making the same false claims. While politicians may refrain themselves from making outright false claims to avoid being fact-checked, oftentimes they even double down after their false claims are debunked. ${ }^{9}$

ClaimPortal leverages the claim matching function in the ClaimBuster API. The fact-check repository is composed of the Share-the-facts 10 fact checks as well as fact checks collected from several fact-checking organizations like PolitiFact, Snopes, factcheck.org, Washington Post, etc. The system measures the similarity between a claim and a fact-check based on the similarity of their tokens. An Elasticsearch server is deployed for searching the repository based on token similarity.

\section{User Interface Features}

ClaimPortal enables a user to sift through the tweets using multiple filters. The important filters are as follows. (1) Keyword search: It allows users to make a text-based search by key-

\footnotetext{
${ }^{9}$ https: / / wapo.st/2rucTq8

${ }^{10}$ http: // www. sharethefacts.org/
}

words such as "climate change". (2) Hashtags: It allows users to further filter tweets by hashtags such as "\#116thCongress" or "\#2020". (3) Claim type: It enables users to search for tweets with a specific claim type, e.g., Conflict or Stance. (4) From: It looks for tweets posted by a particular user handle, e.g., “@ realDonaldTrump”. (5) Mentions: The search results can be filtered further by user mentions (i.e., using "@” to tag a user in a tweet, e.g., “@POTUS”). (6) ClaimBuster score: ClaimPortal also offers a slider to filter results based on a ClaimBuster score range. The result tweets are automatically updated as the slider is moved. (7) Date range: Additionally, the portal offers a date picker to filter tweets based on their creation dates. Figure 2a shows ClaimPortal user interface with the search results of a sample query. The sample query contains the following filtering conditions: a keyword "climate change", a claim type Stance, a range of ClaimBuster score from 0.3 to 1.0 , and a date range from January 1,2019 to April 1, 2019. Moreover, the ClaimPortal shares previously fact-checked claims with users by displaying matching fact-checks after a tweet's card view is clicked at. Figure $2 b$ depicts the matching fact-checks of the highlighted tweet in Figure 2a.

\section{Analytics and Visualizations}

We work to make ClaimPortal the repository where one can find all factual claims made on 
Twitter. It can be a powerful tool for a diverse group of users. It enables web users to explore and analyze factual claims in tweets at scale. We use analytics and visualizations to shed more light on the importance of ClaimPortal and bring the hidden patterns in the data to light. For instance, a user can compare tweets from different political groups in detail based on check-worthiness of their claims and variety of their claims. Figures $3 \mathrm{~d}$ and 3e compare Democratic Senators and Republican Senators based on the types of claims they made and check-worthiness of their claims. Figure 3a depicts the spread of all claim types made by different group of politicians in the past one year and Figure $3 b$ shows the distribution of tweets over five ClaimBuster score ranges made by different group of U.S. politicians such as the 2020 presidential election candidates.

\section{Acknowledgments}

The work is partially supported by NSF grant IIS1719054 and subawards from Duke University as part of a grant to the Duke Tech \& Check Cooperative from the Knight Foundation and Facebook. Any opinions, findings, and conclusions or recommendations expressed in this publication are those of the authors and do not necessarily reflect the views of the funding agencies.

\section{References}

Bill Adair, Mark Stencel, Cathy Clabby, and Chengkai Li. 2019. The human touch in automated factchecking: How people can help algorithms expand the production of accountability journalism. In Computation+Journalism Symposium.

Fatma Arslan, Damian Jimenez, Josue Caraballo, Gensheng Zhang, and Chengkai Li. 2019. Modeling factual claims by frames. In Computation+Journalism Symposium.

Mevan Babakar and Will Moy. 2016. The state of automated fact-checking. Full Fact.

Collin F. Baker, Charles J. Fillmore, and John B. Lowe. 1998. The berkeley framenet project. In $A C L$, pages 86-90.

Giovanni Luca Ciampaglia, Prashant Shiralkar, Luis M. Rocha, Johan Bollen, Filippo Menczer, and Alessandro Flammini. 2015. Computational fact checking from knowledge networks. PLOS ONE, 10:1-13.

Sarah Cohen, Chengkai Li, Jun Yang, and Cong Yu. 2011. Computational journalism: A call to arms to database researchers. In CIDR, pages 148-151.
Naeemul Hassan, Bill Adair, James T. Hamilton, Chengkai Li, Mark Tremayne, Jun Yang, and Cong Yu. 2015a. The quest to automate fact-checking. In Computation+Journalism Symposium.

Naeemul Hassan, Fatma Arslan, Chengkai Li, and Mark Tremayne. 2017a. Toward automated factchecking: Detecting check-worthy factual claims by claimbuster. In $K D D$, pages 1803-1812.

Naeemul Hassan, Chengkai Li, and Mark Tremayne. 2015b. Detecting check-worthy factual claims in presidential debates. In CIKM, pages 1835-1838.

Naeemul Hassan, Gensheng Zhang, Fatma Arslan, Josue Caraballo, Damian Jimenez, Siddhant Gawsane, Shohedul Hasan, Minumol Joseph, Aaditya Kulkarni, Anil Kumar Nayak, Vikas Sable, Chengkai Li, and Mark Tremayne. 2017b. Claimbuster: The first-ever end-to-end fact-checking system. PVLDB, 10(12):1945-1948.

Damian Jimenez and Chengkai Li. 2018. An empirical study on identifying sentences with salient factual statements. In IJCNN.

Saehan Jo, Immanuel Trummer, Weicheng Yu, Xuezhi Wang, Cong Yu, Daniel Liu, and Niyati Mehta. 2019. Verifying text summaries of relational data sets. In SIGMOD.

Chengcheng Shao, Giovanni Luca Ciampaglia, Alessandro Flammini, and Filippo Menczer. 2016. Hoaxy: A platform for tracking online misinformation. In $W W W$ Companion, pages 745-750.

Baoxu Shi and Tim Weninger. 2016. Discriminative predicate path mining for fact checking in knowledge graphs. Knowledge-Based Systems, 104(C):123-133.

Evangelia Spiliopoulou, Eduard Hovy, and Teruko Mitamura. 2017. Event detection using frame-semantic parser. In Proceedings of the Events and Stories in the News Workshop, pages 15-20.

Swabha Swayamdipta, Sam Thomson, Chris Dyer, and Noah A. Smith. 2017. Frame-semantic parsing with softmax-margin segmental rnns and a syntactic scaffold. CoRR, abs/1706.09528.

You Wu, Pankaj K. Agarwal, Chengkai Li, Jun Yang, and Cong Yu. 2017. Computational fact checking through query perturbations. ACM Transactions on Database Systems (TODS), 42(1):4:1-4:41. 